\title{
Ranolazine and Hallucinations
}

\author{
Saminder Singh Kalra ${ }^{\mathrm{a}, \mathrm{c}}$, Zubin Arora $^{\mathrm{b}}$, Craig Nielsen ${ }^{\mathrm{a}}$
}

\begin{abstract}
Ranolazine is a piperazine derivative which produces its anti-ischemic effect by decreasing myocardial oxygen demand and enhancing myocardial perfusion without having any effect on heart rate or blood pressure. Many trials have proved its benefits in the management of chronic stable angina; however, some rare neurological side effects have been seen in elderly population. In this case report, we describe a case of a 75-year-old female with underlying coronary artery disease, chronic kidney disease and mild cognitive impairment who was admitted for a new onset auditory and visual hallucinations along with new onset tremors in both upper and lower extremity after she was started on ranolazine for management of her chronic stable angina. Her workup was negative for any source of infection and no metabolic derangements, drugs or stroke were found. Her symptoms resolved after the ranolazine was discontinued. We recommend starting ranolazine in elderly population only when other anti-ischemic therapies have been exhausted and starting with the lowest therapeutic dose possible. Ranolazine should be discontinued immediately if any signs of neurological adverse effect appear. We also recommend monitoring renal functions in these patients and withholding ranolazine if any signs of worsening renal functions appear.
\end{abstract}

Keywords: Ranolazine; Hallucinations; Tremors; Elderly; Neurological complications

\section{Introduction}

Ranolazine is a piperazine derivative which was approved by FDA in 2006 for the treatment of chronic stable angina refractory to beta and alpha/beta adrenergic blocking agents, calcium channel blockers (CCB) and oral nitrates. Ranolazine produces its anti-ischemic effects by decreasing myocardial oxygen demand and enhancing myocardial oxygen perfusion

Manuscript accepted for publication February 13, 2017

aDepartment of Internal Medicine, The Cleveland Clinic Foundation, Cleveland, OH 44195, USA

bepartment of Gastroenterology and Hepatology, The Cleveland Clinic Foundation, Cleveland, $\mathrm{OH} 44195$, USA

${ }^{\mathrm{c} C}$ Corresponding Author: Saminder Singh Kalra, Department of Internal Medicine, Cleveland Clinic, 9500 Euclid Ave., G10, Cleveland, OH 44195, USA. Email: kalras@ccf.org

doi: https://doi.org/10.14740/jmc2774w while having minimum effect on blood pressure or heart rate $[1,2]$. The beneficial effect of ranolazine in the management of chronic angina has been demonstrated in several trials $[2,3]$. However, there are limited data on the effectiveness of ranolazine in the elderly population, especially those with multiple comorbidities and taking multiple other medications.

In this report, we describe a case of a 75-year-old female who suffered a rare and debilitating neurologic adverse effect after initiation of ranolazine.

\section{Case Report}

A 75-year-old Hispanic female was admitted to the hospital with complaints of new onset visual and auditory hallucinations, and upper and lower extremity tremors for 5 days. Her past medical history included coronary artery disease treated with four-vessel coronary artery bypass graft surgery 15 years ago and a bare metal stent placed 5 years ago, congestive heart disease with last recorded ejection fraction of $30 \%$, stage 4 chronic kidney disease with baseline serum creatinine of $2 \mathrm{mg} /$ $\mathrm{dL}$, mild cognitive impairment and hypertension.

Her home medications included metoprolol $100 \mathrm{mg}$ twice daily, aspirin $325 \mathrm{mg}$ daily, hydralazine $50 \mathrm{mg}$ three times daily, isosorbide dinitrate $20 \mathrm{mg}$ three times daily, furosemide 20 $\mathrm{mg}$ daily, amlodipine $5 \mathrm{mg}$ daily, atorvastatin $40 \mathrm{mg}$ daily and ranolazine $1,000 \mathrm{mg}$ twice daily.

She had been started on ranolazine 6 days prior to admission by her cardiologist for the management of intractable angina.

Upon admission, patient was hemodynamically stable with blood pressure of $92 / 63 \mathrm{~mm} \mathrm{Hg}$, HR of $64 / \mathrm{min}$, temperature $98.6^{\circ} \mathrm{F}$, and oxygen saturation $95 \%$ on room air. She was alert but disoriented to time, place and person and had some difficulty in following commands. Neurological examination revealed coarse resting tremors in her both upper and lower extremities $(1-2 \mathrm{~Hz})$ with no change in tremors with activity. She had normal and equal muscle strength in bilateral upper and lower extremities. She also had normal and equal sensory perception in bilateral upper and lower extremities with no focal neurological deficits.

Initial investigations revealed mild stable anemia with a hemoglobin $10.7 \mathrm{mg} / \mathrm{dL}$ and mean capsular volume (MCV) $91 \mathrm{fL}$ but otherwise normal cell counts including white blood cells $7,620 / \mu \mathrm{L}$ and platelets $251,000 / \mu \mathrm{L}$. Serum chemistry showed acute kidney injury with blood urea nitrogen $50 \mathrm{mg} / \mathrm{dL}$ and serum creatinine $2.84 \mathrm{mg} / \mathrm{dL}$. Other chemistries including blood glucose, serum electrolytes, liver function tests, thyroid 
function tests, vitamin B12 levels and urine toxicology screen were all within normal limits.

Workup to evaluate the cause of her neurological symptoms was performed as follows. Acute coronary syndrome was ruled by three sets of normal cardiac enzymes taken $8 \mathrm{~h}$ apart. Occult infectious etiology was felt unlikely based on normal results of urinalysis, negative urine and blood cultures, and an unremarkable chest X-ray. A computed tomography of brain without contrast showed chronic microvascular white matter changes but with no acute intracranial process. Electroencephalography performed was found to be within normal limits of patient's age with no epileptiform activity. A lumbar puncture was not done given the low clinical suspicion for meningitis (absence of fever, neck rigidity, headache and absence of leukocytosis).

Given the negative results of testing mentioned above, her neurological symptoms were deemed to be secondary to ranolazine toxicity in the setting of acute kidney injury. Therefore, ranolazine was held and her acute kidney injury was treated with careful intravenous hydration. With these measures, her symptoms gradually improved and resolved by day 8 of her hospital stay. Her mental status was noted to be back to baseline following which she was discharged home. She continued to do well and followed up as outpatient 12 weeks after discharge without any recurrence of symptoms.

\section{Discussion}

Chronic stable angina (CSA) or chronic angina pectoris is described as chest pain that occurs during physical exertion or mental/emotional stress and is usually reproducible. Antiischemic therapy has an important role in management of CSA and includes four pharmacological subcategories: beta and alpha/beta adrenergic blocking agents, calcium channel blockers (CCB), oral nitrates and ranolazine [4].

Ranolazine acts by inhibiting the late inward sodium current that is increased in ischemic cardiomyocytes which in turn decreases intracellular calcium load, thus decreasing diastolic tension and oxygen demand [5]. Combination assessment of ranolazine in stable angina (CARISA) trial studied the role of ranolazine on angina frequency and demonstrated a significant higher reduction of mean angina attacks per week and a decrease in average weekly consumption of nitroglycerine by using ranolazine dosage of $750-1,000 \mathrm{mg}$ twice a day compared to placebo [2]. The same results were reproduced by type 2 diabetes evaluation of ranolazine in subjects with chronic stable angina (TERISA) study which evaluated the role of ranolazine in CSA who also had concomitant diabetes [6], Effect of ranolazine in chronic angina (ERIKA) study [3] and metabolic efficiency with ranolazine for less ischemia in non-ST segment elevation acute coronary syndromes (MERLIN-TIMI 36) study [7].

After oral administration, ranolazine reaches its peak plasma concentration between 2 and $5 \mathrm{~h}$. It is primarily metabolized in liver and gut by cytochrome P450 (CYP) 3A4 and to a lesser extent by CYP2D6. Ranolazine reaches its plasma steady state within 3 days of twice daily dosing and is excreted primarily through urine $(75 \%$ as metabolites and $<5 \%$ unchanged drug) [8]. The most common adverse effects reported in clinical trials include dizziness, constipation, nausea, hypotension and headache $[2,3,6,7]$ along with post-marketing reports of angioedema, ataxia, paresthesia, increased serum creatinine and blood urea nitrogen, and torsade de pointes [9].

Case reports of neurological adverse effects from ranolazine have been reported previously. Porhomayon et al reported a case of an elderly lady developing myoclonus after initiation of ranolazine. Myoclonus, in that case, was thought to be as a result of ranolazine's interaction with neuronal sodium channels leading to increased neural sensitivity [10]. Mishra et al described a case of new onset dysarthria, dysmetria and ataxia after initiation of ranolazine therapy in a patient who was being treated with clarithromycin for a lobar pneumonia. The side effect developed due to concomitant use of strong CYP3A inhibitor clarithromycin which increased plasma concentration of ranolazine [11]. Southard et al reported a case of new onset tremors, hallucinations and paresthesia in an elderly female with chronic kidney disease after she was initiated on ranolazine therapy for her chronic angina. The side effect was thought to be from impaired urinary excretion of the drug [12].

In our patient, the time course of initiation of ranolazine and appearance of symptoms, especially in the setting of acute kidney injury, was highly suggestive that the symptoms were related to ranolazine. Also, symptoms improved once ranolazine was held. Additionally, no other causes such as infection, metabolic derangements, drugs, or stroke were found. Although ranolazine has been shown to play a promising role in the management of chronic stable angina, most of the trials excluded the patients with end-stage renal disease on dialysis or patients with glomerular filtration rate of $<30 \mathrm{~mL} / \mathrm{min} / 1.73$ $\mathrm{m}^{2}$. These trials showed no significance difference in safety in patients $>65$ years compared to younger patients, but patients with $>75$ years on ranolazine had a higher incidence of adverse effects $[2,3]$.

To our knowledge, this is the fourth reported case of debilitating neurological symptoms developing after initiation of ranolazine therapy and second reported case of ranolazine causing visual and auditory hallucinations. We recommend that ranolazine be initiated in elderly patients and in patients with chronic kidney disease only after all other medical therapies have been exhausted and at the lowest possible dose of ranolazine especially if the patient is taking other CYP3A inhibitors. Given reports of acute renal failure after initiation of ranolazine, it would be prudent to closely monitor serum creatinine and blood urea nitrogen in these patients so that the medication can be discontinued early if renal function worsens.

\section{Conflicts of Interest}

None.

\section{Source of Funding}

None. 


\section{Disclosures}

None.

\section{References}

1. Chaitman BR, Skettino SL, Parker JO, Hanley P, Meluzin J, Kuch J, Pepine CJ, et al. Anti-ischemic effects and long-term survival during ranolazine monotherapy in patients with chronic severe angina. J Am Coll Cardiol. 2004;43(8):1375-1382.

2. Pepine CJ, Parker JO, Wang W, Wolff AA. Amlodipine, or Diltiazem on Exercise. Cardiology. 2004;291(3):309316.

3. Stone PH, Gratsiansky NA, Blokhin A, Huang IZ, Meng L. Antianginal efficacy of ranolazine when added to treatment with amlodipine: the ERICA (Efficacy of Ranolazine in Chronic Angina) trial. J Am Coll Cardiol. 2006;48(3):566-575.

4. Fox K, Garcia MA, Ardissino D, Buszman P, Camici PG, Crea F, Daly C, et al. Guidelines on the management of stable angina pectoris: executive summary: The Task Force on the Management of Stable Angina Pectoris of the European Society of Cardiology. Eur Heart J. 2006;27(11):1341-1381.

5. Belardinelli L, Shryock JC, Fraser H. Inhibition of the late sodium current as a potential cardioprotective principle: effects of the late sodium current inhibitor ranola- zine. Heart. 2006;92(Suppl 4):iv6-iv14.

6. Kosiborod M, Arnold SV, Spertus JA, McGuire DK, Li Y, Yue P, Ben-Yehuda O, et al. Evaluation of ranolazine in patients with type 2 diabetes mellitus and chronic stable angina: results from the TERISA randomized clinical trial (Type 2 Diabetes Evaluation of Ranolazine in Subjects With Chronic Stable Angina). J Am Coll Cardiol. 2013;61(20):2038-2045.

7. Morrow DA, Scirica BM, Karwatowska-Prokopczuk E, Murphy SA, Budaj A, Varshavsky S, Wolff AA, et al. Effects of ranolazine on recurrent cardiovascular events in patients with non-ST-elevation acute coronary syndromes: the MERLIN-TIMI 36 randomized trial. JAMA. 2007;297(16):1775-1783.

8. Jerling M. Clinical pharmacokinetics of ranolazine. Clin Pharmacokinet. 2006;45(5):469-491.

9. Gilead Sciences. RANEXA ${ }^{\circledR}$ - Prescribing Information. 2016. http://www.gilead.com/ /media/Files/pdfs/medicines/cardiovascular/ranexa/ranexa_pi.ashx.

10. Porhomayon J, Zadeii G, Yarahmadi A, Report C. Case Report A Rare Neurological Complication of Ranolazine. 2013;2013:10-12.

11. Mishra A, Pandya HV, Dave N, Mathew M, Sapre CM, Chaudhary S. A rare debilitating neurological adverse effect of ranolazine due to drug interaction with clarithromycin. Indian J Pharmacol. 2014;46(5):547-548.

12. Southard RA, R MB, Bui AH, Blankstein R. Neurologic adverse effects of ranolazine in an elderly patient with renal impairment. Pharmacotherapy. 2013;33(1):e9-13. 